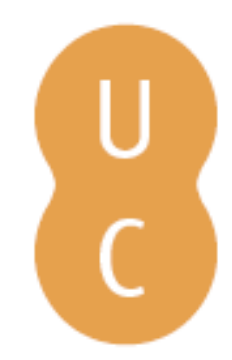

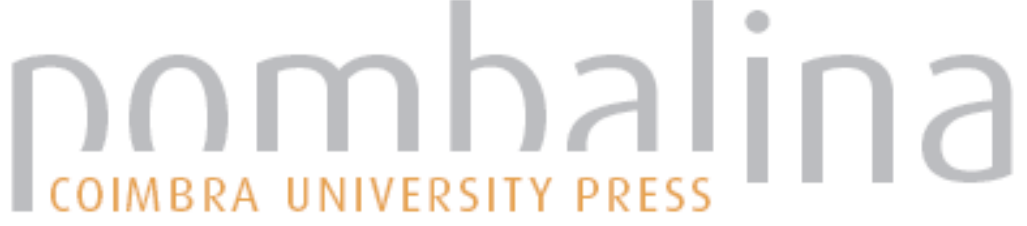

\section{Spontaneous symmetry breaking in quantum physics}

\author{
Autor(es): $\quad$ Providência, J. da
}

Publicado por: Imprensa da Universidade de Coimbra

URL

persistente: URI:http://hdl.handle.net/10316.2/31311

DOI: $\quad$ DOI:http://dx.doi.org/10.14195/978-989-26-0240-0_4

Accessed : $\quad$ 26-Apr-2023 12:49:14

A navegação consulta e descarregamento dos títulos inseridos nas Bibliotecas Digitais UC Digitalis, UC Pombalina e UC Impactum, pressupõem a aceitação plena e sem reservas dos Termos e Condições de Uso destas Bibliotecas Digitais, disponíveis em https://digitalis.uc.pt/pt-pt/termos.

Conforme exposto nos referidos Termos e Condições de Uso, o descarregamento de títulos de acesso restrito requer uma licença válida de autorização devendo o utilizador aceder ao(s) documento(s) a partir de um endereço de IP da instituição detentora da supramencionada licença.

Ao utilizador é apenas permitido o descarregamento para uso pessoal, pelo que o emprego do(s) título(s) descarregado(s) para outro fim, designadamente comercial, carece de autorização do respetivo autor ou editor da obra.

Na medida em que todas as obras da UC Digitalis se encontram protegidas pelo Código do Direito de Autor e Direitos Conexos e demais legislação aplicável, toda a cópia, parcial ou total, deste documento, nos casos em que é legalmente admitida, deverá conter ou fazer-se acompanhar por este aviso.

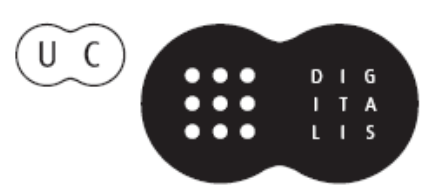


J. S. Redinha

J. da Providência

A. J. C. Varandas

Editors

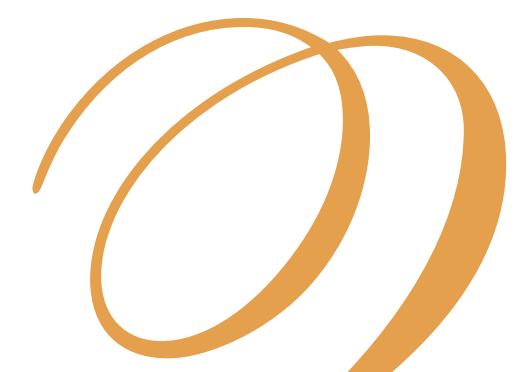

in Chemistry and Physics

A tribute to the memory of

Professor Couceiro da Costa

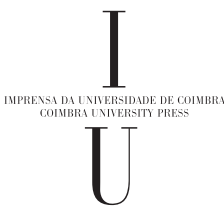




\title{
4. SPONTANEOUS SYMMETRY BREAKING IN QUANTUM PHYSICS ${ }^{1}$
}

\author{
J. da Providência*
}

CFT, Department of Physics, University of Coimbra, Coimbra, Portugal

Following a classification due to Sir Rudolf Peierls, two possible types of spontaneously broken symmetries are discussed, namely, spontaneously broken symmetry of first kind, when a symmetric ground state is degenerate with an asymmetric one, and spontaneously broken symmetry of second kind, when the ground state belongs to a representation of the relevant symmetry group other than the identical one. Symmetry breaking approximation is also considered. In this case, in order to take into account in a simple manner important correlations between the particles of the system, a symmetric ground state of a quantal system is described by an approximation method which violates the symmetry. However, great care should then be exerted in order to insure that the approximation scheme does not break the symmetry to such an extent that the relevant physical properties are distorted. Color superconductivity is an example of such a case which is here discussed in detail.

A novel BCS-type formalism is constructed in the framework of a schematic QCD inspired quark model, having in mind the description of color symmetrical superconducting states. The physical properties of the BCS vacuum (average numbers of quarks of different colors) remain unchanged under an arbitrary color rotation. In the usual approach to color superconductivity, the pairing correlations affect only the quasi-particle states of two colors, the single particle states of the third color remaining unaffected by the pairing correlations. As a consequence, the average numbers of quarks depend apparently on the color, which is an unphysical and undesirable feature. The $S U$ (3) symmetry should not be violated to such an extent. In the theory of color symmetrical superconductivity here proposed, the pairing correlations affect symmetrically the quasi-particle states of the three colors so that vanishing net color-charge is automatically insured. It is found that the ground state energy of the color symmetrical sector of the Bonn model is well approximated by the average energy of the color symmetrical superconducting state proposed here.

\footnotetext{
${ }^{1}$ Talk based on joint work with H. Bohr and C. Providência

*Email address: providencia@teor.fis.uc.pt
} 


\subsection{Introduction}

It is in general expected that the invariance properties of the Hamiltonian of an arbitrary quantum system are shared by its ground state. However, in some situations, this expectation is not realized. We say then that a spontaneous symmetry breaking occurs. Rudolf Peierls [1] refers to spontaneous symmetry breaking of first kind when the ground state is symmetric and is also degenerate, or almost degenerate, with states of a different symmetry. Then, linear combinations of states with different symmetries may arise as a consequence of small perturbations inherent to the processes of preparation or measurement. This is the case of optically active molecules, such as sugar. The two nonstationary asymmetric forms will very slowly transform into one another, in the course of time.

According to Peierls, a broken symmetry of second kind occurs when the ground state belongs to a representation of the relevant symmetry group, other than the identical one. This form of symmetry breaking is present in a ferromagnet which may be regarded as a lattice of $N$ atoms each one possessing a spin $s$, the forces between spins showing a tendency to align them. The total spin is Ns and may point in practically any direction. Thus, the ferromagnet is not isotropic, contrary to the Hamiltonian. Here, the relevant symmetry group is the rotation group of the spin, that is, the group $S U(2)$.

Peierls refers to broken symmetry approximation when, in order to incorporate important correlations, it is convenient to resort to approximations which break some relevant symmetry of the Hamiltonian. This is the case of the shellmodel (a translation leaves the Hamiltonian invariant, but the shell-model breaks this symmetry) and of superconductivity (the global gauge symmetry, which insures the conservation of the number of electrons, is broken by the BCS state). The symmetry is broken by the approximation used to describe the physical state, but not by the physical state itself.

Since the invention of Quantum Chromo-Dynamics (QCD) as the rigorous theory of the strong interaction, it has been generally accepted that quark and gluon fields are the building blocks of nuclear structure, in the framework of 


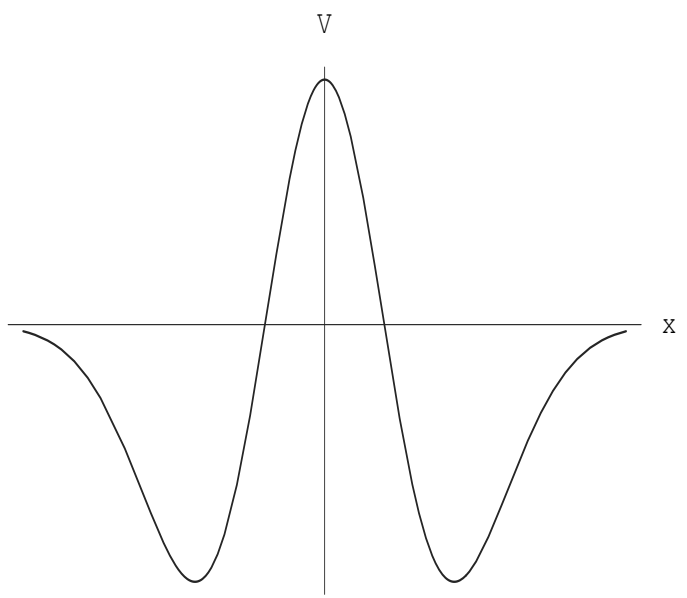

Figure 4.1. The double well potential $V(x)$ in quantum mechanics

QCD field theory. The color quantum number is associated with the SU(3) symmetry which characterizes this theory. The phase equilibrium of hadronic matter is a topic of great current interest of particular relevance to astrophysics. When the density of hadronic matter reaches high values, that is, values which are several times higher than the nuclear density at equilibrium, a great diversity of phases is expected, namely: restoration of chiral symmetry, deconfinement, and color superconductivity. Chiral symmetry is the characteristic symmetry of QCD according to which the helicity of the Dirac fermions with zero mass is a Lorentz invariant. A nucleon is a small cavity, or bag, confining three quarks of different colors. The quark mass is dynamically generated, almost entirely, through a mechanism which is analogous to the Higgs mechanism and is associated with the spontaneous breakdown of chiral symmetry. As the density increases, the nucleonic bags begin to overlap and end up by getting dissolved, so that the quarks become free. Due to the complexity of the theory, it is extremely difficult, if not impossible, to obtain reliable results directly from QCD. It is therefore usual to resort to effective models such as the Nambu-Jona-Lasinio (NJL) model [2-4]. These models assume that the gluonic degrees of freedom produce an attractive two-body force between the quarks, which, effectively, replaces the gluons. Since this force is attractive, it originates the phenomenon of color superconduct- 
ivity, which is analogous to the corresponding superconductivity effect familiar from condensed matter physics and is described as the formation of Cooper pairs, according to the Bardeen-Cooper-Schrieffer (BCS) theory. At present, it is generally accepted that QCD exhibits, at high densities, color superconductivity induced by the phenomenon of Cooper instability. Color superconductivity is supposed to be realized in hadronic matter at extremely high densities, several times the normal nuclear density, which is expected to be found, for instance, in the interior of neutron stars. The color superconducting phase of quark matter, described by the BCS formalism, breaks color symmetry. On the other hand, this symmetry, being a form of gauge symmetry, is not actually broken by the physical state. The breakdown of the $S U(3)$ symmetry by the approximation method is the price one has to pay in order to account for important correlations which are responsible for the superconducting property. However, great care should then be exerted in order to insure that the approximation scheme does not break the symmetry to such an extent that the relevant physical properties are distorted. This fact imposes important restrictions on the BCS formalism which should therefore be used with caution. It is important to identify and discard spurious unphysical states, belonging to representations other than the identical one, which, unavoidably, will artificially emerge.

Effective models have been use with success to investigate the properties of hadronic matter and to obtain a qualitative picture of the phase diagram [5]. Recently, the color superconducting phase has been investigated by many authors in the framework of the BCS approach. Quarks move freely in the deconfined phase, but this phase remains a singlet of the color $S U(3)$ group, since, as remarked, a gauge symmetry can not be spontaneously broken [8].

A BCS state $|\Phi\rangle$ describes a colorless physical state if $N_{1}=N_{2}=N_{3}$, where $N_{i}$ denotes the number of quarks with color $i$, which means that

$$
\left\langle\Phi\left|S_{\lambda_{3}}\right| \Phi\right\rangle=\left\langle\Phi\left|S_{\lambda_{8}}\right| \Phi\right\rangle=0
$$

Here, $S_{\lambda_{k}}$ denotes the $S U(3)$ generator associated with the de Gell-Mann matrix $\lambda_{k}$, that is, $S_{\lambda_{3}} \propto N_{1}-N_{2}, S_{\lambda_{8}} \propto N_{1}+N_{2}-2 N_{3}$. Nevertheless, the condition (4.1) 
is not enough to ensure that $|\Phi\rangle$ is physically acceptable. Since, as observed, the $S U(3)$ symmetry cannot be broken, the physical content of the several states which, by a color rotation, are obtained from a given physically acceptable BCS state, must be the same. Unphysical spurious states, belonging to irreducible representations which are different from the identical one, are invariably exhibited by the effective models which are found in the literature. It is very important to ensure that the performed calculations are not invalidated by those undesirable states. Let $U_{c}$ denote an arbitrary color rotation, i.e., $U_{c}=\exp \sum_{k=1}^{8} i x_{k} S_{\lambda_{k}}$, being $x_{k}$ real and arbitrary parameters. The state $|\Phi\rangle$ must be equivalent to the rotated state $U_{c}|\Phi\rangle$, for any $U_{c}$, as far as expectation values of physical observables are concerned, i.e., the physical properties associated with $|\Phi\rangle$ must be the same as those associated with $U_{c}|\Phi\rangle$. Thus, condition (4.1) must be replaced by condition

$$
\left\langle\Phi\left|U_{c}^{\dagger} S_{\lambda_{3}} U_{c}\right| \Phi\right\rangle=\left\langle\Phi\left|U_{c}^{\dagger} S_{\lambda_{8}} U_{c}\right| \Phi\right\rangle=0
$$

for an arbitrary $U_{c}$, which implies

$$
\left\langle\Phi\left|S_{\lambda_{k}}\right| \Phi\right\rangle=0, \quad k=1,2, \ldots, 8
$$

This is the condition a state $|\Phi\rangle$ must satisfy in order to be physically meaningful. If only condition (4.1) is imposed, and not condition (4.2), the BCS state $|\Phi\rangle$ may not be equivalent to the state $U_{c}|\Phi\rangle$. If this happens, it will belong to an $S U(3)$ representation which is not the identical one, and this is physically unacceptable. In [9], it is shown that the condition (4.2) may be easily implemented. In the next section, the construction of a BCS state which is suitable to describe a color singlet, is illustrated in the framework of a convenient schematic model.

\subsection{A schematic $S U(3)$ pairing model}

At present, it is, in general, admitted that nuclear structure is based on quark and gluon fields, in the framework of QCD field theory. An interesting model (in spite of well known drawbacks) concerning that viewpoint is the Bonn model proposed by Petry et al. [11]. This model describes the nucleus as an MIT bag, 
that is, a set of free quarks bound together by an external pressure. By introducing a decisive pairing force that suppresses unphysical degeneracies of the quark system, many features of nuclear physics are reasonably well accounted for by that model.

The quark model proposed by H. R. Petry et al. [11] is defined by the Hamiltonian

$$
H=G \sum_{j=1}^{3} A_{j}^{\dagger} A_{j}
$$

where

$$
A_{1}^{\dagger}=\sum_{m>0}\left(c_{2 m}^{\dagger} c_{3 \bar{m}}^{\dagger}+c_{2 \bar{m}}^{\dagger} c_{3 m}^{\dagger}\right), \quad \overline{\bar{m}}=m
$$

In Eq. (4.3), $G<0$ is the coupling constant and the expressions for $A_{2}^{\dagger}, A_{3}^{\dagger}$, are obtained by circular permutation of the indices 1, 2, 3. In Eq. (4.4), $c_{i m}^{\dagger}$ are quark creation operators and the indices $i$ and $m$ denote, respectively, the color and the remaining single particle quantum numbers. The state obtained from $m$ by time reversal, in a generalized sense, is denoted by $\bar{m}$.

Color superconductivity has been applied in [9] to the description of the ground state of the Bonn model, which in general is not color symmetric. Indeed, although $H$ has $S U(3)$ symmetry, its eigenstates are not necessarily $S U(3)$ singlets. Since, from the point of view of hadronic physics, the sectors which belong to other representations than the singlet one are not acceptable, the study of the color symmetrical sector is particularly interesting. The generators of color $U(3)$ read

$$
S_{k l}=\sum_{m} c_{k m}^{\dagger} c_{l m}=\sum_{m>0}\left(c_{k m}^{\dagger} c_{l m}+c_{k \bar{m}}^{\dagger} c_{l \bar{m}}\right)
$$

A state $|\Phi\rangle$ is a color singlet if it satisfies the following condition

$$
S_{k l}|\Phi\rangle=0, \quad k \neq l, \quad S_{k k}|\Phi\rangle=\lambda|\Phi\rangle, \quad k=1,2,3 .
$$

Let us consider the general BCS state.

$$
|\Phi\rangle=\exp \sum_{j=0}^{3}\left(K \sum_{0<m \leq \Omega^{\prime}} A_{j m}^{\dagger}+\tilde{K} \sum_{\Omega^{\prime}<m \leq \Omega} A_{j m}\right)\left|0_{\Omega^{\prime}}\right\rangle,
$$


where

$$
\left|0_{\Omega^{\prime}}\right\rangle=\left(\prod_{j=1}^{3} \prod_{\Omega^{\prime}<m \leq \Omega} c_{j m}^{\dagger} c_{j \bar{m}}^{\dagger}\right)|0\rangle,
$$

and

$$
A_{1 m}^{\dagger}=\left(c_{2 m}^{\dagger} c_{3 m}^{\dagger}+c_{2 m}^{\dagger} c_{3 m}^{\dagger}\right) .
$$

The expressions for $A_{2 m}^{\dagger}, A_{3 m}^{\dagger}$, are obtained by circular permutation of the indices $1,2,3$. This state is color neutral in the sense that $\left\langle\Phi\left|\hat{N}_{1}\right| \Phi\right\rangle=\left\langle\Phi\left|\hat{N}_{2}\right| \Phi\right\rangle=$ $\left\langle\Phi\left|\hat{N}_{3}\right| \Phi\right\rangle$, where the quark number operators read

$$
\hat{N}_{j}=\sum_{j} c_{j m}^{\dagger} c_{j m}, \quad j=1,2,3
$$

The parameters $K, \tilde{K}$ are real. We denote by $2 \Omega$ the level degeneracy for a fixed color, that is, the totality of eigenstates pertaining to all quantum numbers beyond color. It is convenient to introduce the notation $\langle W\rangle=\langle\Phi|W| \Phi\rangle /\langle\Phi \mid \Phi\rangle$. If $\Omega^{\prime}=\Omega$, the quark number $N=\left\langle\hat{N}_{1}+\hat{N}_{2}+\hat{N}_{3}\right\rangle$ satisfies $0 \leq N \leq 4 \Omega$. If $\Omega^{\prime}=0$, the quark number $N$ satisfies $4 \Omega \leq N \leq 6 \Omega$. The state vector $|\Phi\rangle$ has obviously zero net color charge, but it is not color symmetrical, that is, it does not fulfil the condition (4.5). However, $K, \tilde{K}$ may be chosen so that $|\Phi\rangle$ is color symmetrical in the average, in the sense that (4.2) is satisfied. We observe that

$$
\begin{aligned}
& c_{1 m}|\Phi\rangle=K\left(c_{2 \bar{m}}^{\dagger}-c_{3 \bar{m}}^{\dagger}\right)|\Phi\rangle, \quad c_{1 \bar{m}}|\Phi\rangle=K\left(c_{2 m}^{\dagger}-c_{3 m}^{\dagger}\right)|\Phi\rangle, \quad 0<m \leq \Omega^{\prime}, \\
& c_{1 m}^{\dagger}|\Phi\rangle=-\tilde{K}\left(c_{2 \bar{m}}-c_{3 \bar{m}}\right)|\Phi\rangle, \quad c_{1 \bar{m}}^{\dagger}|\Phi\rangle=-\tilde{K}\left(c_{2 m}-c_{3 m}\right)|\Phi\rangle, \quad \Omega^{\prime}<m \leq \Omega .
\end{aligned}
$$

These relations are crucial. They are straightforward consequences of the commutation relations

$$
\begin{aligned}
& {\left[c_{1 p},\left(K \sum_{0<m \leq \Omega^{\prime}} A_{j m}^{\dagger}+\tilde{K} \sum_{\Omega^{\prime}<m \leq \Omega} A_{j m}\right)\right]=K\left(c_{2 \bar{p}}^{\dagger}-c_{3 \bar{p}}^{\dagger}\right), \quad 0<p \leq \Omega^{\prime},} \\
& {\left[c_{1 p}^{\dagger},\left(K \sum_{0<m \leq \Omega^{\prime}} A_{j m}^{\dagger}+\tilde{K} \sum_{\Omega^{\prime}<m \leq \Omega} A_{j m}\right)\right]=-\tilde{K}\left(c_{2 \bar{p}}-c_{3 \bar{p}}\right), \quad \Omega^{\prime}<p \leq \Omega .}
\end{aligned}
$$

From (4.6) it follows that the BCS vacuum $|\Phi\rangle$ is annihilated by the operators

$$
\begin{array}{lll}
d_{1 m}=c_{1 m}-K\left(c_{2 \bar{m}}^{\dagger}-c_{3 \bar{m}}^{\dagger}\right), & d_{1 \bar{m}}=c_{1 \bar{m}}-K\left(c_{2 m}^{\dagger}-c_{3 m}^{\dagger}\right), & 0<m \leq \Omega^{\prime}, \\
d_{1 m}=c_{1 m}^{\dagger}+\tilde{K}\left(c_{2 \bar{m}}-c_{3 \bar{m}}\right), & d_{1 \bar{m}}=c_{1 \bar{m}}^{\dagger}+\tilde{K}\left(c_{2 m}-c_{3 m}\right), & \Omega^{\prime}<m \leq \Omega .
\end{array}
$$


The expressions for $d_{2 m}, d_{3 m}, d_{2 \bar{m}}, d_{3 \bar{m}}$, are obtained by circular permutation of the indices 1, 2, 3. These operators characterize the so-called Bogoliubov quasiparticles. The transformation in Eq. (4.7) is not canonical, since $\left\{d_{i m}, d_{j m}^{\dagger}\right\} \neq \delta_{i j}$, but the corresponding canonical transformation, which is not needed for the present purpose, may be easily obtained. We observe that the contractions $\left\langle c_{i m}^{\dagger} c_{j m}\right\rangle, i \neq j$, are independent of $i, j$. Similarly the contractions $\left\langle c_{j m}^{\dagger} c_{j m}\right\rangle$, are independent of $j$.

If $0<m \leq \Omega^{\prime}$, we easily find that

$$
\left\langle c_{i m}^{\dagger} c_{j m}\right\rangle=\left\langle c_{i \bar{m}}^{\dagger} c_{j \bar{m}}\right\rangle=-\frac{K^{2}}{1+3 K^{2}}, i \neq j, \quad\left\langle c_{j m}^{\dagger} c_{j m}\right\rangle=\left\langle c_{j \bar{m}}^{\dagger} c_{j \bar{m}}\right\rangle=\frac{2 K^{2}}{1+3 K^{2}} .
$$

If, on the other hand, $\Omega^{\prime}<m \leq \Omega$, we have

$$
\left\langle c_{i m}^{\dagger} c_{j m}\right\rangle=\left\langle c_{i \bar{m}}^{\dagger} c_{j \bar{m}}\right\rangle=\frac{\tilde{K}^{2}}{1+3 \tilde{K}^{2}}, \quad i \neq j, \quad\left\langle c_{j m}^{\dagger} c_{j m}\right\rangle=\left\langle c_{j \bar{m}}^{\dagger} c_{j \bar{m}}\right\rangle=1-\frac{2 \tilde{K}^{2}}{1+3 \tilde{K}^{2}} .
$$

Thus, we obtain

$$
\left\langle S_{i j}\right\rangle=-2 \Omega^{\prime} \frac{K^{2}}{1+3 K^{2}}+2\left(\Omega-\Omega^{\prime}\right) \frac{\tilde{K}^{2}}{1+3 \tilde{K}^{2}}, \quad i \neq j .
$$

By conveniently choosing $K, \tilde{K}$, we may insure that Eq. (4.2) is satisfied, so that the BCS vacuum $|\Phi\rangle$ represents a color singlet. We recall that the $S U(3)$ generators $S_{\lambda_{j}}$ associated with the Gell-Mann matrices $\lambda_{j}$ read

$$
\begin{array}{lll}
S_{\lambda_{1}}=S_{21}+S_{12}, & S_{\lambda_{2}}=i\left(S_{21}-S_{12}\right), & S_{\lambda_{3}}=S_{11}-S_{22}, \\
S_{\lambda_{4}}=S_{31}+S_{13}, & S_{\lambda_{5}}=i\left(S_{31}-S_{13}\right), & S_{\lambda_{6}}=S_{32}+S_{23}, \\
S_{\lambda_{7}}=i\left(S_{32}-S_{23}\right), & S_{\lambda_{8}}=\frac{1}{\sqrt{3}}\left(S_{11}+S_{22}-2 S_{33}\right) . &
\end{array}
$$

The state $|\Phi\rangle$ satisfies automatically (4.1), which is very convenient, but is not sufficient. Indeed, (4.1) remains valid when we replace $|\Phi\rangle$ by $U_{c}|\Phi\rangle$, for an arbitrary color rotation $U_{c}$, only if (4.2) is further implemented.

Next we compute the contractions $\left\langle c_{2 m} c_{1 \bar{m}}\right\rangle=\left\langle c_{3 m} c_{2 \bar{m}}\right\rangle=\left\langle c_{1 m} c_{3 \bar{m}}\right\rangle=$ $\left\langle c_{2} \bar{m} c_{1 m}\right\rangle=\left\langle c_{3 \bar{m}} c_{2 m}\right\rangle=\left\langle c_{1 \bar{m}} c_{3 m}\right\rangle=: D_{m}$, where $D_{m}$ is real. We find

$$
D_{m}=\frac{K}{1+3 K^{2}}, \quad \text { for } \quad 0<m \leq \Omega^{\prime} ; \quad D_{m}=\frac{\tilde{K}}{1+3 \tilde{K}^{2}}, \quad \text { for } \quad \Omega^{\prime}<m \leq \Omega .
$$


We are now able to compute the energy expectation value

$$
\frac{\mathcal{E}}{G}=\sum_{j=1}^{3} \frac{\left\langle\Phi\left|A_{j}^{\dagger} A_{j}\right| \Phi\right\rangle}{\langle\Phi \mid \Phi\rangle} .
$$

Let $p=\Omega^{\prime} / \Omega, q=1-p=\left(\Omega-\Omega^{\prime}\right) / \Omega$. Define $\theta$, $\tilde{\theta}$ such that $\sqrt{3} K / \sqrt{1+3 K^{2}}=$ $\sin \theta, 1 / \sqrt{1+3 K^{2}}=\cos \theta, \sqrt{3} \tilde{K} / \sqrt{1+3 \tilde{K}^{2}}=\sin \tilde{\theta}, 1 / \sqrt{1+3 \tilde{K}^{2}}=\cos \tilde{\theta}$. Then, having in mind (4.8), the color symmetry constraint $\left\langle S_{i j}\right\rangle=0, i \neq j$, reduces to

$$
p \cos 2 \theta-q \cos 2 \tilde{\theta}=p-q \text {. }
$$

The main contribution to the energy expectation value comes from the square of the expectation values $\left\langle A_{1}\right\rangle=\left\langle A_{2}\right\rangle=\left\langle A_{3}\right\rangle=\Omega(p \sin 2 \theta+q \sin 2 \tilde{\theta}) / \sqrt{3}$, which involve contractions of the form $\langle c c\rangle$, The corresponding constrained extremum occurs for $\cos 2 \theta=-\cos 2 \tilde{\theta}=p-q, \sin 2 \theta=\sin 2 \tilde{\theta}=\sqrt{1-(p-q)^{2}}$, so that, in the leading order, we have

$$
\frac{\mathcal{E}}{G} \approx \Omega^{2}\left(1-(p-q)^{2}\right)
$$

In terms of the variables $\theta, \tilde{\theta}$, the number of quarks reads

$$
N=6 \Omega\left[p \frac{1-\cos 2 \theta}{3}+q\left(1-\frac{1-\cos 2 \tilde{\theta}}{3}\right)\right] \text {. }
$$

At the extremum, $N=6 \Omega q$, and

$$
\frac{\mathcal{E}}{G} \approx \Omega^{2}\left[1-\left(1-\frac{N}{3 \Omega}\right)^{2}\right] .
$$

To complete the calculation of $\mathcal{E} / G$ we must add the small corrections coming from the neglected contractions of the form $\left\langle c^{\dagger} c\right\rangle$.

In terms of $\theta, \tilde{\theta}$, we have

$$
\begin{array}{ll}
\left\langle c_{j m}^{\dagger} c_{j m}\right\rangle=\left\langle c_{j m}^{\dagger} c_{j m}\right\rangle=\frac{1}{3}(1-\cos 2 \theta), & \text { for } \quad 0<m \leq \Omega^{\prime}, \\
\left\langle c_{j m}^{\dagger} c_{j m}\right\rangle=\left\langle c_{j m}^{\dagger} c_{j m}\right\rangle=1-\frac{1}{3}(1-\cos 2 \tilde{\theta}), & \text { for } \quad \Omega^{\prime}<m \leq \Omega .
\end{array}
$$

At the extremum, $\left\langle c_{j m}^{\dagger} c_{j m}\right\rangle=\left\langle c_{j m}^{\dagger} c_{j m}\right\rangle=2 q / 3$, for $0<m \leq \Omega^{\prime},\left\langle c_{j m}^{\dagger} c_{j m}\right\rangle=$ $\left\langle c_{j \bar{m}}^{\dagger} c_{j m}\right\rangle=(1+2 q) / 3$, for $\Omega^{\prime}<m \leq \Omega$. Finally, the ground state energy of the color symmetrical super-conducting phase reads,

$$
\frac{\mathcal{E}}{G}=\frac{N}{9}\left(6 \Omega-N+1+\frac{4 N}{3 \Omega}\right), \quad 0 \leq N \leq 6 \Omega .
$$




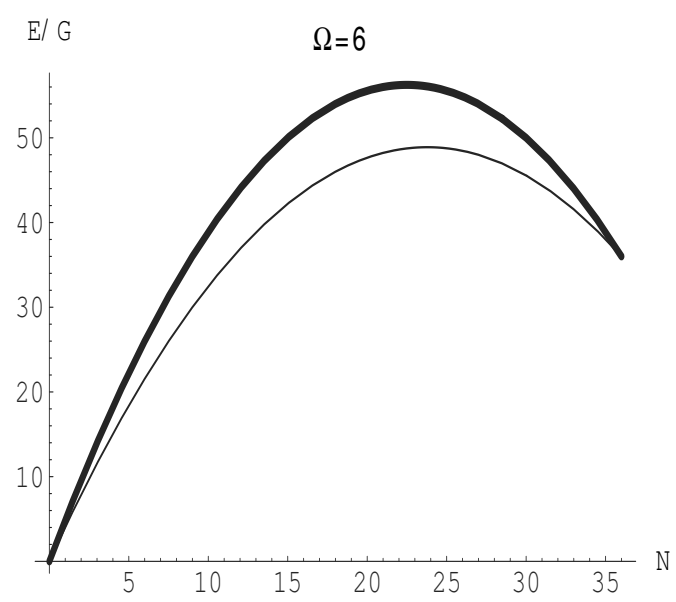

Figure 4.2. ground state energy of the color symmetrical sector versus the quark number, for $\Omega=6$. Thick line: exact result according to (4.10); thin line: color symmetrical BCS estimate according to (4.9). Since $G<0$, the upper curve lies below in energy, in agreement with Ritz theorem.

Using the Schwinger type representation of $s u(4)$, formulated in terms of appropriate boson operators, which was developed by Yamamura et al. [12], the color symmetrical sector of the Bonn model has been characterized in [13] There, the exact ground state energy of the color symmetrical sector was found to read

$$
\frac{\mathcal{E}}{G}=\frac{N}{3}\left(2 \Omega+3-\frac{N}{3}\right), \quad 0 \leq N \leq 6 \Omega .
$$

It is interesting to compare Eqs. (4.9) and (4.10). This is done, in Figure 4.2.

\subsection{Conclusions}

Following a classification due to Sir Rudolf Peierls, two possible types of spontaneously broken symmetries have been discussed, namely, spontaneously broken symmetry of first kind, when a symmetric ground state is degenerate with an asymmetric one, and spontaneously broken symmetry of second kind, when the ground state belongs to a representation of the relevant symmetry group other than the identical one. Symmetry breaking approximation may also be considered. In this case, in order to take into account in a simple manner important correlations, a symmetric ground state of a quantal system is described 
by an approximation method which violates the symmetry. However, great care should then be exerted in order to insure that the approximation scheme does not break the symmetry to such an extent that physical properties are distorted. Color superconductivity is an example of such a case which is here discussed in detail.

We have constructed a BCS-type formalism, based on a conveniently generalized Bogoliubov transformation, which is appropriate to describe color symmetrical superconducting states of quark matter. It is found that the ground state energy of the color symmetrical sector of the Bonn model is well approximated by the average energy of the color symmetrical superconducting state proposed here and it is easily seen that the color symmetric BCS result becomes closer to the exact one while $\Omega$ increases.

It should be emphasized that the present approach automatically ensures vanishing net color charge, even if (4.2) is not imposed. Condition (4.2) is important because most field theoretic models contain sectors which belong to other color $S U(3)$ representations than the color singlet one. Such sectors are therefore unphysical and should be discarded.

\section{References}

[1] R. Peierls, J. Phys. A: Math. Gen. 24, 5273 (1991).

[2] Y. Nambu, G. Jona-Lasinio, Phys. Rev. 122, 345 (1961); Phys. Rev. 124, 246 (1961).

[3] S. P. Klevanky, Rev. Mod. Phys. 64, 649 (1992).

[4] T. Hatsuda and T. Kunihiro, Phys. Rep. 247, 221 (1994).

[5] M. Buballa, Phys. Rep. 407, 205 (2005).

[6] M. Alford, K. Rajagopal and F. Wilczek, Phys. Lett. B 422247 (1998); Nucl. Phys. B 537, 443 (1999).

[7] M. G. Alford, K. Rajagopal, T. Shaefer, A. Schmitt, Rev. Mod. Phys. 80, 1455 (2008).

[8] S. Elitzur, Phys. Rev. D 12, 3978 (1975).

[9] H. Bohr and J. da Providência, J. Phys. A: Math. Theor. 41, 405202 (2008). 
[10] H. Bohr, C. Providência and J. da Providência, Eur. Phys. J. A 41, 355 (2009); Eur. Phys. Lett. 89, 42001 (2010).

[11] H. R. Petry, H. Hofstaedt, S. Merk, K. Bleuler, H. Bohr and K. S. Narain, Phys. Lett. B 159, 363 (1985).

[12] M. Yamamura, T. Kuriyama, A. Kunihiro, Prog. Theor. Phys. 104, 385 (2000).

[13] C. Providência, J. da Providência, F. Cordeiro, M. Yamamura, S. Nishiyama and Y. Tsue, Ann. Phys. 324, 1666 (2009). 